\title{
Successful lung transplantation after prone positioning in an ineligible donor: a case report
}

\author{
Eunjeong Son ${ }^{1} \cdot$ Jinook Jang ${ }^{1} \cdot$ Woo Hyun $\mathrm{Cho}^{1} \cdot$ Dohyung Kim ${ }^{2} \cdot$ Hye Ju Yeo ${ }^{1,3}$ (D)
}

Received: 2 May 2021 / Accepted: 18 June 2021 / Published online: 22 June 2021

(c) The Japanese Association for Thoracic Surgery 2021

\begin{abstract}
Atelectasis is a reversible factor in hypoxemia among brain-dead donors. In ineligible donors, prone positioning reverses atelectasis and improves oxygenation. We present a successful lung transplantation after salvaging a previously unviable lung. A 37-year-old woman presented with acute pontine hemorrhage that progressed to brain death. The initial partial pressure of oxygen $\left(\mathrm{PaO}_{2}\right)$ /fraction of inspired oxygen $\left(\mathrm{FiO}_{2}\right)$ ratio of the donor's lung was $342 \mathrm{mmHg}$. The $\mathrm{PaO}_{2} / \mathrm{FiO}_{2}$ ratio dropped to $49 \mathrm{mmHg}$ due to atelectasis. There was no improvement despite recruitment maneuvers, bronchoscopy, and chest percussion. After placing the donor in the prone position for four hours, electrical impedance tomography showed improved atelectasis. The donor did not experience hemodynamic instability. The lung was transplanted into a patient with Kartagener's syndrome with situs inversus. The surgical procedure was uneventful. He was successfully weaned from the mechanical ventilator on the second-day post-transplantation and was discharged from the hospital after 4 weeks.
\end{abstract}

Keywords Ineligible donor $\cdot$ Brain-dead donors $\cdot$ Prone position $\cdot$ Lung transplantation $\cdot$ Kartagener syndrome

\section{Introduction}

The demand for lung transplantation has increased recently, and the shortage of lung donors is a critical issue [1]. Annually, approximately $20 \%$ of anticipatory lung transplant patients worldwide die on the waiting list [2,3]. Although the use of marginal donors has increased in response to this problem, several donor lungs are not used due to poor lung conditions [3]. One of the main reasons why donor's lungs

Eunjeong Son and Jinook Jang contributed equally to this manuscript.

Hye Ju Yeo

dugpwn@naver.com

1 Department of Internal Medicine, Division of Pulmonary, Allergy, and Critical Care Medicine, Pusan National University Yangsan Hospital, Yangsan, Republic of Korea

2 Department of Thoracic and Cardiovascular Surgery, Pusan National University Yangsan Hospital, Yangsan, Republic of Korea

3 Department of Pulmonology and Critical Care Medicine, Research Institute for Convergence of Biomedical Science and Technology, Pusan National University Yangsan Hospital, Geumo-ro 20, Beomeo-ri, Mulgeum-eup, Yangsan, Gyeongsangnam 626-770, Republic of Korea are not used is the low oxygen $\left(\mathrm{PaO}_{2}\right)$ /fraction of inspired oxygen $\left(\mathrm{FiO}_{2}\right)$ ratio [4]. In brain-dead (BD) donors, atelectasis can occur due to the absence of cough and respiratory drive, resulting in ventilation-perfusion (V/Q) mismatch and hypoxemia [5]. Additionally, individual donor factors, especially risk factors such as mechanical ventilation, supine positioning, and obesity can lead to atelectasis [6]. Aggressive lung management protocols that incorporate recruitment maneuvers, bronchoscopy, chest percussion, and lung-protective ventilation can be utilized to reverse the atelectasis and make donor lungs more suitable for transplantation [7]. However, in cases with no response to this conventional approach, prone positioning may be a viable alternative. We present a case of successful lung transplantation after salvaging a previously unviable lung via prone positioning.

\section{Case}

A 37-year-old woman presented with an acute pontine hemorrhage that progressed to BD. Her medical history was unremarkable, and she was a non-smoker. She was obese with a body mass index of $29 \mathrm{~kg} / \mathrm{m}^{2}$. She was chosen to be a lung donor with a $\mathrm{PaO}_{2} / \mathrm{FiO}_{2}$ ratio of $342 \mathrm{mmHg}$, the margin for donation. The next day, the $\mathrm{PaO}_{2}$ dropped to $49 \mathrm{mmHg}$ 
on a $\mathrm{FiO}_{2}$ of 1.0, and chest radiography demonstrated progressive right lower lobe infiltrates, suggestive of atelectasis (Fig. 1a). Bronchoscopy showed full obstruction of both lower lobes by purulent sputum (Fig. 1b). The lung became unviable for donation. Despite aggressive lung management, including recruitment maneuvers, bronchoscopy, and chest percussion, there was no improvement in the $\mathrm{PaO}_{2} /$ $\mathrm{FiO}_{2}$ ratio. The donor was placed in the prone position for $19 \mathrm{~h}$, while continuing the routine treatments. After being in the prone position for four hours, electrical impedance tomography (EIT) showed improved atelectasis (Fig. 2a). The $\mathrm{PaO}_{2} / \mathrm{FiO}_{2}$ ratio significantly increased (Fig. 3), and atelectasis disappeared on radiography (Fig. 2b). The donor did not experience significant hemodynamic instability or adverse events while in the prone position. The donor's lung was transplanted into a 54-year-old man with Kartagener's syndrome accompanied by situs inversus. At the time of procurement, the lung showed good compliance and mild edema in the dependent position of the bilateral lower lobe. The surgical procedure was uneventful, and primary graft dysfunction did not occur. Arterial blood gas analysis of the recipient, which was performed as soon as possible after the lung transplantation, showed $\mathrm{PaO}_{2}$ of $637 \mathrm{mmHg}$ on a $\mathrm{FiO}_{2}$ of 1.0. He was successfully weaned from the mechanical ventilator on the second day of transplantation and was discharged from the hospital after 4 weeks.

\section{Discussion}

This case showed that ventilation in the prone position of an ineligible donor lung reversed the hypoxemia and atelectasis. This ultimately resulted in a more viable transplant. The prone position acutely improved oxygenation in a hypoxemic $\mathrm{BD}$ donor with atelectasis without adverse events. However, in BD donors, if there is severe hemodynamic instability or a possibility that the organ to be transplanted can be damaged by abdominal wounds or multiple trauma, the prone position is not suitable. A careful approach is required depending on the donor's condition [8].
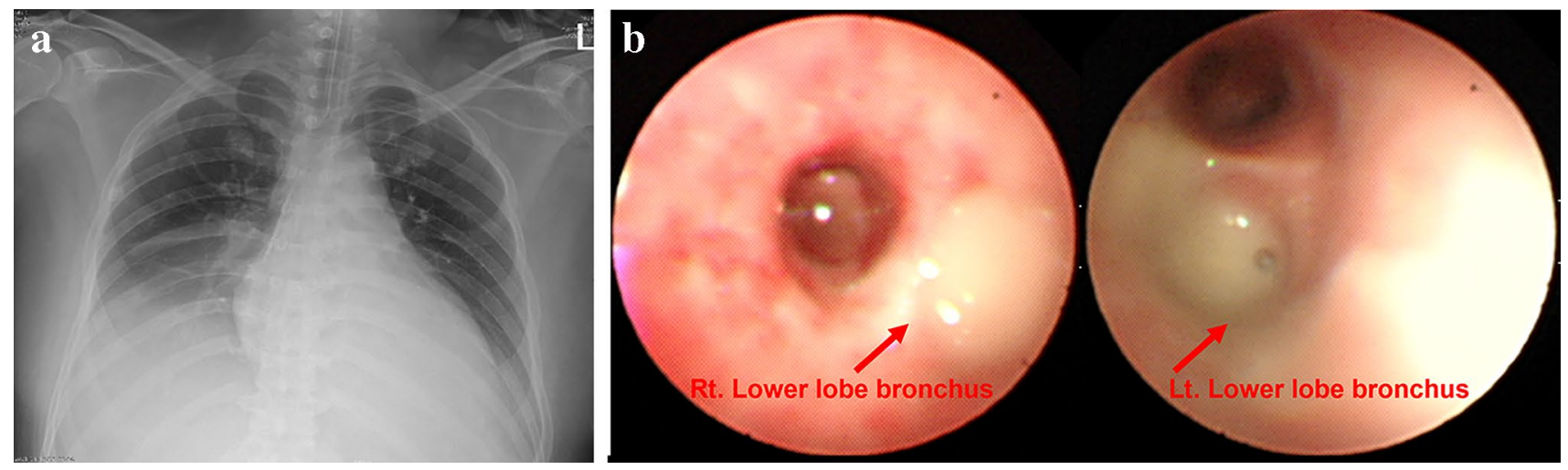

Fig. 1 Images of donor lungs before prone position. a Chest radiograph, b Bronchoscopy

a

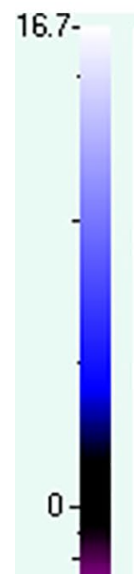

b

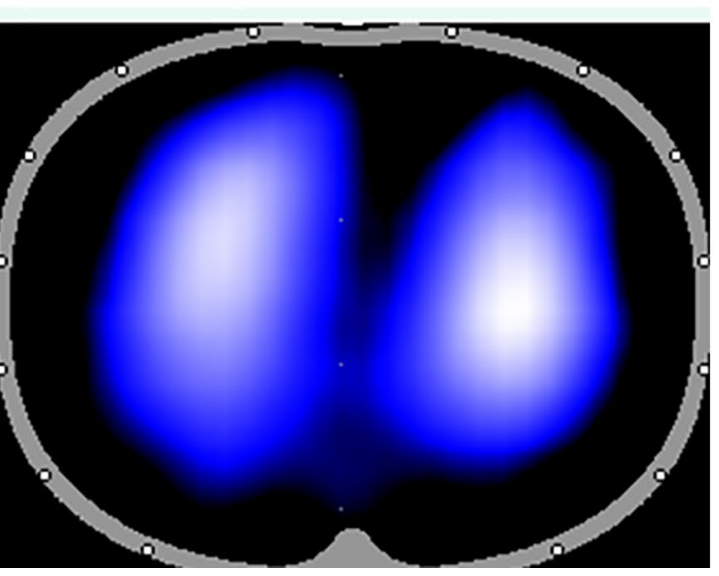

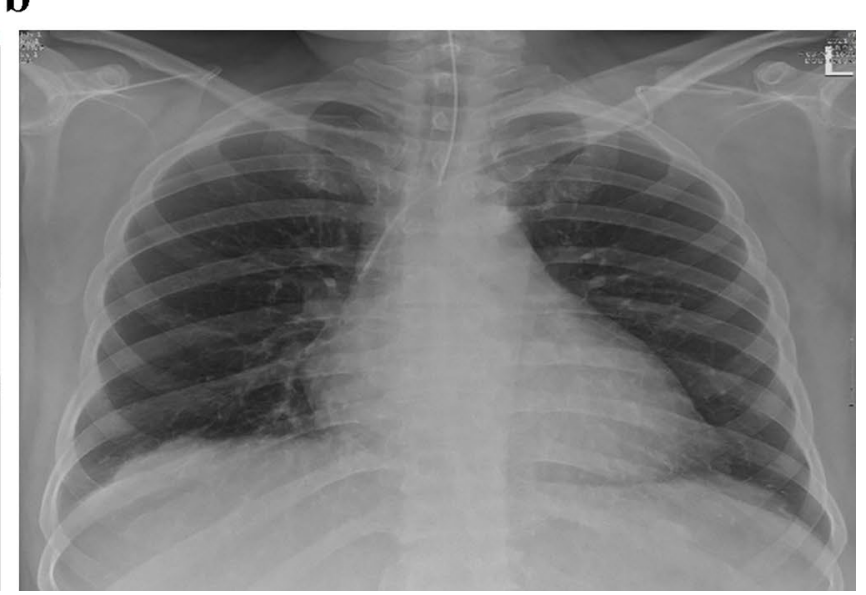

Fig. 2 Images of donor lungs after prone position. a Electrical impedance tomography, b Chest radiograph 
Fig. 3 Changes of $\mathrm{PaO}_{2} / \mathrm{FiO}_{2}$ ratio
$-\square-\mathrm{PaO} 2 / \mathrm{FiO} 2$ ratio

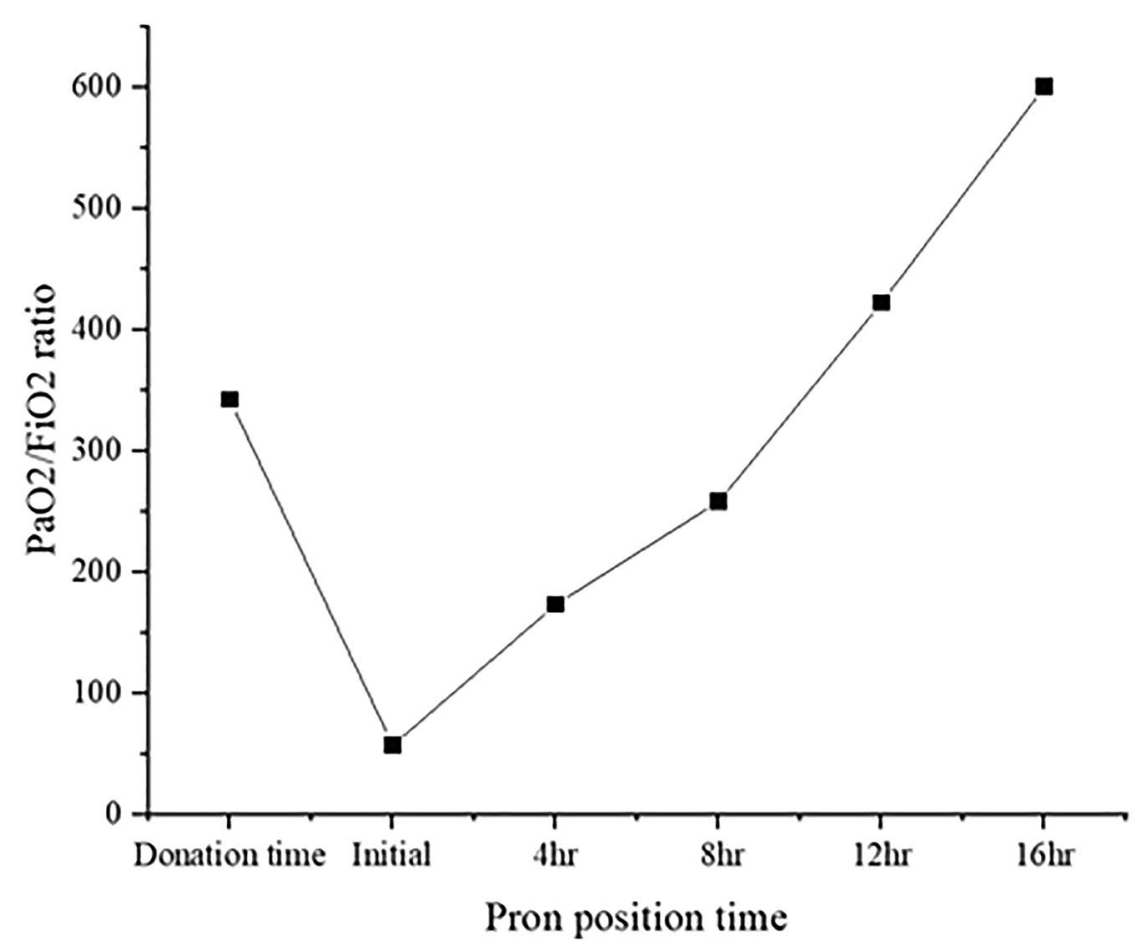

Atelectasis is a correctable problem that is common among donors. In a recent study, atelectasis was found in $45 \%$ of donors using chest computed tomography (CT) [9]. The lungs of BD donors are easily obstructed due to excessive secretions, atelectasis, infection, inflammation, and gravitational forces, which result in a ventilation-perfusion mismatch and dead space ventilation [10]. For marginal or unsuitable donors, aggressive lung management protocols can improve oxygenation and allow lung transplantation [11]. In this case, the donor's hypoxemia was not corrected by the conventional recruitment maneuver. It returned to a viable state after the donor was in the prone position for $12 \mathrm{~h}$. Traditionally, the prone position has been used for acute respiratory distress syndrome [12]. It allows the alveoli to open and participate in gas exchange, which increases $\mathrm{PaO}_{2}$ [8]. Recently, Marklin et al. suggested the effect of prone positioning in BD donors. The reaction to prone positioning started to occur after four hours, and a significant improvement in oxygenation was observed after $12 \mathrm{~h}$, as seen in this case [7]. The improved oxygenation due to prone positioning is likely mediated by rapid positional improvements in the V/Q mismatch, enhanced postural drainage of secretions, and sustained improvements in atelectasis and shunting [7, 8, 12]. In this case, we confirmed the reversal of the non-aerated region with EIT instead of CT. As EIT is a non-invasive monitoring method that makes cross-sectional images of the chest through the analysis of transthoracic electrical conductivity, this technique is radiation-free and applicable at the bedside. It is used for the evaluation of regional lung ventilation instead of CT in situations where it is difficult to conduct CT as in our case [13].

Currently, there are concerns about the development of complications or difficulties in evaluating other organs while the donor is in the prone position. The prone position can be associated with a variety of complications, many of which are derived from increased pressure on anterior structures [14]. Inappropriate pressure on vital structures of the abdomen can cause ischemia and organ failure [15]. Obese donors, such as in this case, are at particular risk of increased abdominal pressure, increased venous pressure in the head, decreased cardiac output, and reduced end-organ blood flow. However, with proper supportive padding, these donors have better oxygenation in the supported prone position, as mediastinal contents fall anteriorly facilitating ventilation. In a previous study, the only adverse event related to the prone position was arterial line dislodgment; there were no inadvertent extubations [8]. In our case, no complications occurred. In terms of evaluating other organs, the heart was first evaluated at the supine position, and the second evaluation was performed when the donor was changed from the prone position to the supine position again. There was no difficulty in evaluating the heart and liver. In the same context, other studies have shown that there was no difference in the utilization of the heart, liver, and kidney with the donor in the prone and supine positions $[4,7]$. 
The mortality on the waiting list has been high because of the imbalance between the number of available organs and potential recipients [3]. Therefore, it is necessary to increase the number of available lungs for transplantation through active lung management of marginal or unacceptable donors [8]. Although prone positioning has been widely accepted in the intensive care unit for refractory hypoxemia, it is still a novel treatment in lung donor management. This case showed that prone positioning salvaged marginal or unacceptable donor lungs with presumptive atelectasis refractory to conventional management. It is a feasible technique to improve donor lung function.

\section{Conclusion}

Prone positioning acutely improved the oxygenation of a hypoxemic BD donor's lungs with atelectasis. This was a safe and critical solution that improved the lung condition of a marginal or unacceptable lung and enabled transplantation.

\section{References}

1. Chaney J, Suzuki Y, Cantu E III, van Berkel V. Lung donor selection criteria. J Thorac Dis. 2014;6(8):1032.

2. Valapour M, Lehr CJ, Skeans MA, Smith JM, Carrico R, Uccellini K, et al. OPTN/SRTR 2016 annual data report: lung. Am J Transplant. 2018;18(Suppl 1):363-433.

3. Yeo HJ, Kim DH, Kim YS, Jeon D, Cho WH. Performance changes following the revision of organ allocation system of lung transplant: analysis of Korean network for organ sharing data. J Korean Med Sci. 2021. https://doi.org/10.3346/jkms.2021.36.e79.

4. Marklin G, O'Sullivan C, Dhar R. Prone ventilation in brain-dead organ donors acutely increases oxygenation and results in more lungs transplanted. J Heart Lung Transplant. 2020;39(4):S375.

5. Okamoto T, Niikawa H, Wheeler D, Ayyat KS, Basem S, Itoda $\mathrm{Y}$, et al. Selective recruitment of large lower lobe atelectasis on donor back table in rejected donor lungs. Transplant Direct. 2019;5(5):e453.
6. Chang SH, Kreisel D, Marklin GF, Cook L, Hachem R, Kozower BD, et al. Lung focused resuscitation at a specialized donor care facility improves lung procurement rates. Ann Thorac Surg. 2018;105(5):1531-6.

7. Marklin GF, O'Sullivan C, Dhar R. Ventilation in the prone position improves oxygenation and results in more lungs being transplanted from organ donors with hypoxemia and atelectasis. J Heart Lung Transplant. 2021;40(2):120-7.

8. Mendez MA, Fesmire AJ, Johnson SS, Neel DR, Markham LE, Olson JC, et al. A 360 degrees rotational positioning protocol of organ donors may increase lungs available for transplantation. Crit Care Med. 2019;47(8):1058-64.

9. Gauthier JM, Bierhals AJ, Liu J, Balsara KR, Frederiksen C, Gremminger E, et al. Chest computed tomography imaging improves potential lung donor assessment. J Thorac Cardiovasc Surg. 2019;157(4):1711-8.

10. Copeland H, Hayanga JWA, Neyrinck A, MacDonald P, Dellgren $\mathrm{G}$, Bertolotti A, et al. Donor heart and lung procurement: a consensus statement. J Heart Lung Transplant. 2020;39(6):501-17.

11. Lerg G, Shanta L. Intrapulmonary percussive ventilation as a lung recruitment strategy in brain-dead organ donors. Prog Transplant. 2017;27(1):84-9.

12. Jang YE, Ji SH, Kim EH, Lee JH, Kim JT, Kim HS. Effect of regular alveolar recruitment on intraoperative atelectasis in paediatric patients ventilated in the prone position: a randomised controlled trial. Br J Anaesth. 2020;124(5):648-55.

13. Steinmann D, Stahl CA, Minner J, Schumann S, Loop T, Kirschbaum A, et al. Electrical impedance tomography to confirm correct placement of double-lumen tube: a feasibility study. Br J Anaesth. 2008;101(3):411-8.

14. Kwee MM, Ho YH, Rozen WM. The prone position during surgery and its complications: a systematic review and evidencebased guidelines. Int Surg. 2015;100(2):292-303.

15. Chikhani M, Evans DL, Blatcher AW, Jackson AP, Guha IN, Aithal GP, et al. The effect of prone positioning with surgical bolsters on liver blood flow in healthy volunteers. Anaesthesia. 2016;71(5):550-5.

Publisher's Note Springer Nature remains neutral with regard to jurisdictional claims in published maps and institutional affiliations. 